DOSSIÊ TEMÁTICO: Vitalidade do sujeito e poder de formação: narrativas autobiográficas em diálogo

\title{
CLAROSCURO DE LA TRANSMISIÓN, ACOMPAÑAMIENTO Y FORMACIÓN DE PROFESORES NÓVELES
}

\author{
CHIAROSCURO OF THE TRANSMISSION, ACCOMPANIMENT AND BILDUNG OF \\ NOVEL TEACHERS
}

\author{
O CLARO-ESCURO DA TRANSMISAO, ACOMPANHAMENTO E FORMAÇÃO DE \\ PROFESSORES INICIAIS
}

\author{
José Antonio Serrano Castañeda \\ Universidad Pedagógica Nacional-Ajusco - México \\ Noemí Hernández Suárez \\ Universidad Pedagógica Nacional-Ajusco - México \\ Juan Mario Ramos Morales \\ Universidad Pedagógica Nacional-Ajusco - México
}

\begin{abstract}
Resumo: $\mathrm{O}$ artigo reflete sobre os elementos que caracterizam a noção de transmissão no processo de acompanhamento de docentes recém-iniciados em ambientes institucionais de ensino superior. Inscrevese no projeto de investigação Processos curriculares e práticas de acompanhamento (PCyPA). A formação é um processo cultural que se dá em condições materiais e com estratégias de organização dos laços sociais. No tempo e no espaço do ato cultural, desencadeiam-se os múltiplos vínculos humanos que organizam a trama, o drama da transmissão como ato de mediação entre o que é apresentado pelos especialistas e o que se incorpora nos recém-chegados às práticas institucionais. O encontro entre dois agentes - geracionalmente distintos - assume a forma de disposições para construir trajectos que conduzam à mobilização do saber - especialmente o desejo de saber - e de querer ser de uma determinada forma que conduz à modelagem de si, ao formação de si mesmo a partir da força vital que se localiza na transmissão. O processo de incorporação está do lado da transformação, modificação, alteração, quase conversão. A transmissão não deixa de ter efeitos comunicacionais, mas não se reduz a isso. A transmissão tem efeitos políticos porque dá origem a uma forma de organizar e projetar os laços sociais, tende a gerar uma estética - como princípio da realidade - e a cultivar estratégias colegiadas e quadros coletivos. Para refletir sobre o claro-escuro da transmissão, tomamos dois diários narrativos de professoras construídos no processo de imersão em uma instituição de ensino superior; de um lado, expõem o processo de se tornarem professores universitários, de outro, revelam as vicissitudes do acompanhamento: hesitações, paixões, interesse e formação de si.
\end{abstract}

Palavras-chave: Acompanhamento. Formação de professores. Transmissão. 
José Antonio Serrano Castañeda | Noemí Hernández Suárez | Juan Mario Ramos

\begin{abstract}
This article is about the elements around the notion of transmission in the process of accompanying teachers who have just started work in institutional settings of higher education. The work is part of the research project Curricular processes and accompanying practices (PCyPA). Training is a cultural process that takes place under material conditions and with strategies for organizing social ties. In the time and space of the cultural act, multiple human links are unleashed that organize the plot, the drama of the transmission as an act of mediation between what is presented by the experts and what is incorporated in the newcomers to institutional practices. The encounter between two agents generationally different - takes the form of dispositions to build paths that lead to the mobilization of knowledge - especially the desire to know - and of wanting to be in a certain way that leads to the modeling of the self, to the formation of oneself from the vital force that is located in the transmission. The incorporation process is on the side of transformation, modification, alteration, almost conversion. Transmission does not stop having communicational effects, but it is not reduced to it. Transmission has political effects since it gives way to organize and project social ties it tends to generate an aesthetic as a principle of reality - and to cultivate collegiate strategies and collective frameworks. To reflect on the chiaroscuro of the transmission, we took two narrative diaries of teachers built in the process of immersion in a higher education institution. On the one hand, they expose the process of becoming university teachers; on the other, they reveal the vicissitudes of the accompaniment: hesitations, passions, interest and shaping of the self.
\end{abstract}

Keywords: Accompaniment. Bildung. Transmission

Resumen: El artículo reflexiona sobre los elementos que caracterizan a la noción de transmisión en el proceso de acompañar a profesores que recién inician labores en marcos institucionales de educación superior. Se inscribe en el proyecto de investigación Procesos curriculares y prácticas de acompañamiento. Formar es un proceso cultural que se efectúa en condiciones materiales y con estrategias de organización de los vínculos sociales. En el tiempo y espacio del acto cultural se desencadenan múltiples vínculos humanos que organizan la trama, el drama de la transmisión en tanto acto de mediación entre lo que se presenta por los expertos y lo que se incorpora en los recién ingresados a las prácticas institucionales. El encuentro entre dos agentes — generacionalmente distintos - toma la forma de disposiciones para construir trayectos que llevan a la movilización del saber —en especial del deseo de saber - y del querer ser de una determinada manera que conduce a la modelación del yo, a la formación de sí a partir de la fuerza vital que se localiza en la transmisión. El proceso de incorporación está del lado de la transformación, de la modificación, de la alteración, casi de la conversión. La transmisión no deja de tener efectos comunicacionales, pero no se reduce a ella. La transmisión tiene efectos políticos pues da cauce a una forma de organizar y proyectar los vínculos sociales, tiende a la generación de una estética — en tanto principio de realidad - y al cultivo de estrategias colegiadas y marcos colectivos. Para reflexionar sobre los claroscuros de la transmisión tomamos dos diarios narrativos de profesoras construidos en el proceso de inmersión a una institución de educación superior; por un lado, exponen el proceso de convertirse en docentes universitarios, por otro, revelan los avatares del acompañamiento: vacilaciones, pasiones, interés y moldeamiento del yo.

Palabras clave: Acompañamiento, Formación de profesores, Transmisión.

\title{
Introducción
}

Las universidades se comprometen con la mejora integral de la docencia, la génesis de conocimiento a través de actividades de investigación, la difusión y extensión académica. Se configuran como un espacio en el que se ponen en juego relaciones sociales —con diversos 
efectos- entre los agentes educativos participantes (docentes, estudiantes, personal administrativo). Sujetos en juego - que han incorporado el mundo social y cultural que los envuelve - frente a la normativa académica y a las particularidades de los campos profesionales.

No basta con los conocimientos operativos y conceptuales que los profesionales recién egresados han adquirido sobre un campo disciplinar específico y los procesos de enseñanzaaprendizaje básicos para impartir una clase. La universidad demanda a los futuros profesores familiarizarse con distintos tipos de práctica educativa, de prácticas sociales que habitan en una institución de educación superior. En el juego institucional (BOURDIEU, 1995), los profesores con experiencia han aprendido las normas y costumbres más relevantes de su hacer cotidiano; han incorporado las reglas del juego y el habitus de la institución; conocen las filias y fobias del espacio académico; aspectos que el profesor novel (e inexperto) desconoce y que podrían frustrar el inicio de su actividad como personal de recién ingreso a la institución.

Un grupo de profesores de la Universidad Pedagógica Nacional (UPN) han promovido —bajo el proyecto Proceso curriculares y prácticas de acompañamiento- desde 2017 un proceso de formación continua a dos profesoras novel a través de prácticas de acompañamiento por parte de dos profesores con experiencia. Éstos asumen el rol de mentores, pues «se sitúan aparte de las normas institucionales, constituyendo, por tanto, un importante estímulo para la innovación y la creatividad. En consecuencia, los mentores tienen la posibilidad de convertirse en poderosos agentes de cambio en la enseñanza superior» (BROCKBANK y McGILL, 2008, p. 282).

Con la finalidad de develar, por un lado, los retos a los que se enfrentan los profesores noveles; por otro, los efectos que las prácticas de acompañamiento tienen sobre ellos; y los desafíos que los procesos de este calibre conllevan, recuperamos la experiencia de dos profesoras que ingresaron, respectivamente, en 2017 y 2018 a laborar como docente a la UPN. Dado que las funciones universitarias son diversificadas, en el texto se relatan el proceso de acompañamiento relativo a la docencia. Las otras funciones requieren otras modalidades de presentación de la experiencia de acompañamiento.

\section{Hilvanes del acompañamiento institucional}

Dentro del proyecto Proceso curriculares y prácticas de acompañamiento los profesores con experiencia han asumido el compromiso de acompañar a las profesoras novel a partir de la reflexión de la práctica. Los profesores con experiencia no solo modelan - en 
presencia y a la distancia - modos de ser o hacer que han interiorizado a lo largo de su carrera institucional y que las novel aún desconocen y requieren incorporar (saberes, habilidades, normas); también facilitan y fomentan un trabajo de reflexión sobre la acción y en la acción, es decir, se convierten cada uno en un «formador reflexivo»(PERRENOUD, 2001, p.64).

Las prácticas de acompañamiento se basan, fundamentalmente, en un diálogo permanente crítico y reflexivo en el que ambas partes - los profesores con experiencia y las profesoras noveles - comparten inquietudes, dificultades, alternativas, disyuntivas, consejos, cuestionamientos; sentidos de la experiencia. En palabras de Perrenoud «una práctica reflexiva profesional jamás es completamente solitaria. Se apoya en conversaciones informales, en momentos organizados de profesionalización interactiva» (2001, p. 192). Tanto los profesores con experiencia como las profesoras noveles aspiran a vincularse desde una posición horizontal y simétrica, a pesar de reconocer la diferencia en el saber al uso disponible en las actividades institucionales (SCHÖN, 1992).

Los profesores con experiencia y las profesoras noveles escriben un diario como dispositivo de reflexión y fuente de experiencias. Al relatar el sentido de la experiencia se plantean preguntas, trazan las dificultades a las que se ha enfrentado, proyectan sus expectativas, deseos y sentimientos, plantean propuestas, confrontan teorías, etc. El diario permite a los profesores reconstruir, comprender y otorgar un nuevo sentido a su práctica; les da la oportunidad de distanciarse y observarse a sí mismos. De este modo, los profesores con experiencia pueden dar seguimiento a pesar de no estar físicamente; proporcionar retroalimentación; recomendar rutas de acción; proponer formas de conceptualizar la experiencia. Al momento, las profesoras noveles tienen la posibilidad de retornar a sí mismo, de recuperar elementos que lo apoyen a transformar su práctica desde una posición crítica y consciente.

A partir de la sistematización de experiencias en el diario, los profesores — novel y con experiencia - están en condiciones de indagar, analizar, comprender, develar sentidos, mantener una relación reflexiva con lo que viven; hacer consciencia de sus posturas éticas, profesionales, políticas, pedagógicas y morales frente a lo que hacen; mirarse desde ángulos distintos. En palabras de Contreras, en el diario se narra, pero «el relato no es una transcripción de lo vivido, sino una composición movida por la inquietud que lo vivido me suscitó» (2016, p. 20). Así, los profesores documentan, reflexionan y crean nuevos significados. 


\section{La experiencia de transmisión}

Recurrimos a la perspectiva biográfico-narrativa desde dos lógicas distintas, intrínsecamente enlazadas. Por un lado, es desde esta tradición que trabajamos la escritura de un diario con características específicas; tiene como objetivo que las profesoras novel y los docentes con experiencia estén en condiciones de desplegar la práctica reflexiva. Por otro lado, dejar al descubierto cómo es que las profesoras novel elaboran e incorporan, le dan sentido a la experiencia de afiliación a la institución.

El encuentro institucional es entre sujetos. Los sujetos, en tanto agentes sociales son individuos históricos, dotados-dotadores de sentido a la vida individual producto de la vida en común. El agente es un ser activo, intérprete, reflexivo, recreador de su devenir en el mundo en articulación con el «otro», que el interaccionismo simbólico denominó el «otro generalizado» (la cultural, el lenguaje y los ritos) (SERRANO, 2016), Bajtin la «dialéctica autor-destinatario», y Gadamer «la anticipación del otro» (RUFINETTI, 2013; TODOROV, 1995). Para dar cuenta de lo que acontece en el nudo de la relación yo-tú, las ciencias sociales construyen una jerga variada que va desde el lazo social, vínculo, apego, transferencia, relación, influencia, transmisión (MUNNÉ, 1995). En su conjunto engarzadas en la perspectiva que hemos asumido de experiencia (DEWEY, 2008; PORTA y RAMALLO, 2013; SERRANO, RAMOS 2017).

La experiencia no se reduce a los sentidos, están incluidos; la experiencia no es la base de un conocimiento más elevado, es una forma de ser del saber; la experiencia no es fundamentalmente cognoscitiva, interpela al ser en su conjunto. Expresado en sentido positivo, la experiencia es testimonio de la incorporación cualitativa del entorno circundante (personas, cosas, valores, códigos). El sentido de la experiencia es el logro de nuestro andar en el mundo como transacción entre el actor y el entorno.

La experiencia es acción, es hacer y es padecer - recibir activamente, lo que otros hacen. Es un padecer activo pues en el intercambio con los otros el agente está en posibilidad de elaborar el significado del hacer. Dice Dewey que «La materia prima de la experiencia necesita ser reelaborada» (2008, p. 85). La creación del sentido - momentáneo, contextual y listo para ser reelaborado - es el papel de la reflexión. Los efectos de la reflexión exponen socialmente las variaciones de sentido de lo experimentado. «El significado... no es nunca simple o unidimensional, sino que es multidimensional y estratificado» (VAN MANEN, 2003, p. 96). En la vida diaria se pueden experimentar variados asuntos, es experiencia cuando se crea sentido sobre ella, cuando actúan dosis de reflexión. La reflexión surge del encuentro cualitativo 
con el mundo y da dirección a nuestro vivir que «implica selección, elección y decisión» (BERNSTEIN, 2010, p. 136).

Cabe señalar que el asunto de la imaginación es central en la obra de Dewey, afirma «La imaginación es el medio de apreciación en todos los campos»(DEWEY, 1998, p. 202). Más adelante advierte, «Por la imaginación es por la que los símbolos se traducen en su significado directo y se integran con una actividad más limitada para ampliarla y enriquecerla» (DEWEY, 1998, p. 203). Para Dewey, la imaginación es una vía de transformación social. La imaginación está vinculada al futuro de la acción, es anticipación, es posibilidad en un tiempo por venir. En la resolución de problemas la imaginación es despliegue de potencialidades. Es la vía para preparar nuevas relaciones con el mundo y nuevas vías de la acción sobre el mundo

La experiencia se construye a partir de lo ya sabido, de lo ya confeccionado, lo ya imaginado, de la estructura de sentido que se cimienta en el transitar por la vida; lo nuevo entra en un fondo de imaginación. Por esta vía, la experiencia fundada es la que nutre el sentido de inquirir y llevar hacia nuevas experiencias, de indagar, de confeccionar modos diversos de saber sobre el mundo; experiencia y deseo de saber van de la mano. La experiencia es un esfuerzo por cambiar lo que ha sido dado; impulsa la indagación — ir hacia lo desconocido—-, abre hacia lo nuevo, a lo que está por configurarse, o experimentarse a futuro. En nuestra vida diaria la experiencia es actual, presente, engarzada a lo ya experimentado, al pasado que preludia ir hacia adelante. Es irrepetible, efectuada en la incertidumbre y particular pues se logra en contextos concretos.

En esta serie de reflexiones, cabe enlazar la noción de transmisión — como experiencia de transmisión - para comprender el proceso de acompañamiento en la inmersión de los profesores novel por los experimentados. La transmisión es asunto de todo vínculo humano (particulariza el tema en las prácticas institucionales que tienen como finalidad formar al otro a partir de procesos particulares) de traspaso de saber, pasar la estafeta en marcos institucionales. Particular proceso que en el vocabulario pedagógico se torna en nociones tales como educación, enseñanza, aprendizaje, el vínculo educativo, la relación pedagógica, entre otros. Simultáneamente, la transmisión es asunto de los vínculos intergeneracionales, como sería el caso del proceso de acompañamiento entre novel y experimentado.

Se enlazan en la transmisión: las condiciones, el saber, lo imaginario, la illusio. Unas palabras sobre cada uno de estos componentes de la transmisión. En la transmisión — como vasos comunicantes - ceden su lugar el mundo objetivo y subjetivo. En el mundo simbólico la transmisión es un proceso intersubjetivo con buenas dosis de intrasubjetividad. La transmisión es posible dado que sobre nuestras espaldas pesa la historia de la humanidad que ha 
confeccionado dos procesos inherentes en las condiciones de la transmisión: la materia organizada y la organización materializada.

Del lado de las condiciones de la transmisión está la dialéctica entre la materia organizada y organización materializada. La materia organizada concentra la permanencia material que hace susceptible el proceso de organización de la comunicación de la transmisión: la estrategia. Incluye los

[...] modos semióticos (el tipo de signo utilizado: texto, imagen o sonido), del dispositivo de difusión (lineal, radial, interconectado) del soporte físico (piedra, madera, papiro, papel, ondas), así como los medios de transporte de los hombres y de los mensajes (caminos, vehículos, infraestructuras, redes, etcétera). (DEBRAY, 1997, p.28 las cursivas son del autor)

Del lado de la organización materializada — la institución con su logística — está el saber sabido. El saber institucionalizado de los grupos sociales que proyectan su sentido —el oxígeno que da proyección a su existencia (TODOROV, 1995) - a fin de concretar variados modos de cohesión entre los grupos. Para nuestro caso, el trasvase del saber académico es lo que permite a los actores - en tanto funcionan como grupo- distinguirse frente a otros significantes (grupos, temas de evocación, modos de organización de los ritos institucionales). La organización materializada está en los sistemas culturales que disponen "configuraciones comunitarias, a saber, las diversas formas de cohesión que unen a los operadores humanos de una transmisión (o más exactamente, impuestas a ellos por la naturaleza material de los signos y dispositivos utilizables)" (DEBRAY, 1997, p.29, las cursivas son del autor).

En segundo lugar, lo que está en juego en la transmisión es el saber en sus diversas modalidades (saber; ser estar, hacer, tácito, sabido, inconsciente, preconsciente, de la vida cotidiana, proposicional, institucional, deseo de saber, por mencionar algunos). El saber, al ser diversificado y abarcar una multitud de objetos e interpretaciones relativas a ellos, es imposible de ser trasmitido en su forma total. En el proceso de transmisión el saber está dosificado; además, en las dosis del saber al otro en todo momento hay un resto que se encuentra fuera de la transmisión. El saber se transmite en fragmentos y nunca se cierra; el saber está siempre abierto; hacer público el saber es la punta de lanza para la edificación de otro tipo de saber.

La transmisión no se reduce al saber cognitivo, proposicional —organizado en redes semánticas, con jerga específica engendrada en las disciplinas para dar cuenta de sus objetos. En el acto de transmisión se anuda un espectro amplio de saberes encarnados en los actores. El cuerpo sabe, habla con una gramática a ser interpretada por los actores en el cumplimiento del rol de cara a otro rol. Particulariza al saber estar ligado a las tradiciones de grupos, campos; 
por otro lado, el saber es la fuente $-\mathrm{y}$ producto- de diálogos inter e intrasubjetivos (SERRANO; RAMOS, 2017).

Un aspecto más en la transmisión (algunos rasgos han sido presentados líneas arriba) es la imaginación, «ya sea mítica, filosófica o científica, establece el pacto entre el espíritu y la naturaleza. Sin ella no existirían los mundos simbólicos que han inspirado a los artistas y a los hombres de ciencias» (ARNAU, 2020, p. 11). El pragmatismo proporciona una visión de la imaginación como acto creativo: la acción es acción creativa. La creatividad de la acción lleva a «la comprensión del modo en que se relacionan los entramados valorativos, los elementos normativos, la comprensión de nosotros mismos, de los demás y del mundo en el horizonte de la acción»(LEYVA; KOZLAREK, 2002, p. 19). Desde este lugar, la imaginación creativa se despliega en contextos situados, concretos, particulares; se pone en acto porque anticipa la solución a la acción, tiene en la mira la esperanza y la eventualidad de construir significado de las diversas vetas que producen los problemas del día a día. En la imaginación creativa - la intuición, en tanto saber-sabido- el saber preestablecido se pone acto en la edificación del sentido.

La transmisión es inherente a la actividad humana, pero las estrategias que se ponen en acto son locales; está ligada a lo que Bourdieu denominó campos, «Cada campo define y activa una forma específica de interés, una illusio específica como reconocimiento tácito del valor de las apuestas propuestas en el juego y como dominio práctico de las reglas que lo rigen» (BOURDIEU, 1995, p. 80). La illusio describe el saber estratégico que se forja en los grupos localizados, saber producto de la acción práctica y a ella dirigida. La illusio ponen en acto los intereses de los agentes sociales en el proceso de transmisión. «Numerosos modos de pensamiento y de acción... se transmiten de la práctica a la práctica, mediante modos de transmisión totales y prácticos basados en el contacto directo y duradero entre quien enseña y quien aprende ("Haz lo mismo que yo")» (BOURDIEU, 1995, p. 164). A pesar de que la transmisión es un asunto social, inherentes al conjunto de prácticas en el espacio social, adquiere matices especiales en cada uno de los campos sociales, como lo es el de las instituciones educativas de nivel superior, de formación de profesionales de la educación.

\section{El diario, reflexionar la experiencia de transmisión}

Existen diversos modos para recoger el mundo intersubjetivo de los sujetos: historia de vida, diario, trayectoria de vida, carpeta de aprendizaje, entrevista, biografía. En todos ellos, el sujeto relata las experiencias que lo han formado y transformado, «la experiencia se constituye 
de esta relación entre lo que nos pasa y el significado que atribuimos a lo que nos afectó. Esto se hace mediante el acto de narrar y de (re) interpretar» (DA CONCEIÇÃO, 2015, p.78).

La narrativa permite al sujeto revelar, organizar, reconstruir y dar sentido a las experiencias (con efectos cognitivos, emocionales, de re-significación, transformación y actualización); recuperar el pasado, acoger el presente y proyectar pautas en el futuro. Por lo que refiere Delory-Momberger «La dinámica de la narrativa encadena los acontecimientos, entre una situación inicial de inocencia e inexperiencia y una situación terminal de madurez y domino, según una causalidad final que invita a una lectura retrospectiva» (2009, p. 51).

Desde esta lógica, a través de la escritura de sus diarios, las profesoras novel y los docentes experimentados dan forma al encuentro (real e imaginario) que afecta a su historia de vida No hay que olvidar que la escritura de un relato nos obliga, irremediablemente, a reformar nuestros propios pensamientos en torno a lo acaecido, a reflexionar. Es proceso reflexivo el que nos guía a reelaborar, reconstruir y transformar nuestro estar en el mundo. La figura del sujeto

[...] no es algo dado cuya existencia podríamos constatar, demostrando su estado, sino una construcción siempre en el acto, o sea, un conjunto dinámico de operaciones, un proceso. El sujeto no cesa de instituirse como sujeto: él es el objeto incesante de su propia institución. (DELORY-MOMBERGER, 2009, p.104)

La narrativa permite recuperar la singularidad e irrepetibilidad de las experiencias; producir saber, en este caso, sobre las prácticas de acompañamiento y la formación de los sujetos que en ellas intervienen (CONELLY, Y CLANDININ, 1995). La perspectiva narrativa conduce a develar las relaciones, tensiones, afiliaciones, fobias, filias y resistencias acaecidas en las profesoras noveles y los docentes con experiencia; comprender cómo aquellos viven la docencia, la mentoría, lo imprevisto, cómo son afectados o padecidos. Si bien, en las investigaciones narrativas, el investigador recupera la voz del sujeto y la organiza a través de criterios de objetivación, el sujeto estudiado se convierte en co-investigador de su propia vida (BOLÍVAR, DOMÍNGUEZ Y FERNÁNDEZ, 2001, p. 36), en autor de su historia.

A continuación, recuperamos la voz de dos profesoras novel que han vivido el acompañamiento por parte de dos profesores con experiencia. Recuperamos fragmentos de cada uno de los diarios que han trabajado desde que se incorporaron a la plantilla docente de la Universidad Pedagógica Nacional. Los relatos nos ofrecen la posibilidad de mostrar las relaciones entre los sujetos y las fuerzas del espacio académico; las posiciones ocupadas sucesivamente dentro de éste (BOURDIEU, 1997), así como, «indagar en lo que vivimos y nos 
José Antonio Serrano Castañeda | Noemí Hernández Suárez | Juan Mario Ramos

pasa para obtener una mayor consciencia, percepción, comprensión de los acontecimientos que vivimos» (CONTRERAS, 2016, p. 16).

\section{Particularidades del acompañamiento. Encuentros y desencuentros}

El proceso de acompañamiento que han vivido las profesoras noveles se conforma por tres momentos clave: inmersión, acompañamiento y autonomía asistida (en marcos temporales corresponden al ciclo escolar de un periodo escolar, para nuestro caso semestre).

En la primera fase —inmersión-, el profesor novel se sumerge al espacio académico. Acompaña como codocente al profesor con experiencia responsable del grupo, quien modela modos de acción dentro del salón de clases y transmite saberes propios del quehacer del docente en una institución universitaria (tomar en cuenta en la docencia a los planes y programas de estudio, revisar los reglamentos institucionales dinámicas normativas y proporcionar pistas sobre los procesos administrativos normados o al uso). El profesor novel asume el compromiso de sistematizar su experiencia en un diario (instrumento de trabajo en el que da cuenta de la trayectoria académica, retos, fortalezas, intereses, cuestionamientos, prejuicios, ideas) que le permite movilizar su experiencia - el sentido de los conocimientos y saberes previos- para dotar de nuevos sentidos a su práctica. Cabe advertir que las profesoras noveles eligieron sus seudónimos.

Un fragmento del diario de Prusia (profesora novel que ingresó en 2018 a la UPN), advierte:

J. M. se ha convertido en mi mentor en el ámbito universitario. No siempre me dice qué y cómo hacer mi trabajo, sino que lo observo, escucho y tomo notas de aquellos aspectos que me resultan relevantes de retomar en las sesiones. Cuando no lo hago, él me cuestiona: “ya tomó nota?". Me impresiona el estilo "tan" diplomático que tiene para tratar diversos asuntos, no solo relacionados con la enseñanza, también con cuestiones administrativas e institucionales ("de grande, quiero ser como él"). Él me ha insistido en involucrarme en trámites administrativos - los cuales repelo- con el propósito de que me familiarice con los procesos institucionales de la universidad. Me ha explicado que debo aprender cómo están las cosas al interior del área para que no siempre necesite de él. Por ejemplo, tuve que familiarizarme con 1) el plan de estudios de la licenciatura en Administración Educativa; 2) las dinámicas y normas institucionales; 3) los procesos de gestión de convenios inter institucionales para el espacio de prácticas; 4) el fundamento del proyecto de Prácticas Profesionales de Gestión Cultural, de la Formación Continua y Recursos Tecnológicos, del que soy parte ahora, así como 5) los modos, tendencias, filias y manías de la UPN, en especial, del área Académica 1. (2019, p.6)

La profesora Oliva (quien ingresó a la UPN en 2017) dilucida: 
El acompañamiento que tuve de ambos [J.M. y J.A.] profesores en su calidad como experimentados fortaleció mi seguridad de pararme frente a los estudiantes. El hecho de que en ocasiones me acompañaran a las sesiones, fue también para mí motivo para poner atención a su manera de hablar, de conducirse, de posicionarse: con autoridad, con claridad de las cosas, con la exteriorización en voz alta de las expectativas hacia los estudiantes. Decirles de manera precisa, concisa - con ejemplos - a los alumnos lo que esperamos de ellos, lo que pueden hacer en diversos escenarios y apoyarles a ampliar su mirada y campo de acción - desde la presentación de trabajo o entrega de tareas - me motivó a reflexionar sobre los sentidos que la formación universitaria tiene para mí. (2019, p.7)

En lo que se refiere a la segunda etapa —acompañamiento-, el profesor novel pasa a ser el responsable del grupo en el siguiente semestre (grupo diferente al del primer momento) y coordina las clases; mientras que el profesor con experiencia ocupa el lugar de codocente e interviene solo para puntualizar aspectos que el novel exceptúa. Olivia (2019) narra cómo ha sido el trabajo en colaboración entre ella y el profesor con experiencia, su mentor:

La manera en que nos hemos acoplado para el trabajo dentro y fuera de clase es a través de la comunicación, retroalimentación y reflexión constante. Él tiene la facilidad de aportar ideas, textos, estructura para el curso. Las conversamos y, a partir de mis comentarios, las retroalimentamos; las construimos en conjunto. Admiro su capacidad para pensar en todo momento. Libros, películas o experiencias que él tiene las traslada para la formación de los estudiantes de alguna u otra manera. Me invita a indagar, leer, escribir, estar en actividad constante y conversar de lo que podemos hacer. Dentro del aula, ambos aportamos, en mayor medida él —a comparación mía-. (2019, p. 9-10)

Al respecto, Prusia comparte sobre esta segunda fase.

De nuevo, mi mentor me legitimó — con su actitud y acciones - como una persona capaz de dar seguimiento a las prácticas de los estudiantes. A pesar de que no siempre estuvo presente en las sesiones - a veces llegaba la última hora-, siempre estuvo al tanto de lo que hacía; solíamos vernos antes de las sesiones para "estar en sintonía" y evitar contradecirnos; para acordar a quién le tocaba abordar ciertos temas; había algunos que por mi inexperiencia era complicado que pudiese resolverlos. Creo que nos acoplamos bien; estuvimos en el mismo canal; ambos nos apoyamos. Además, reconozco que, de mi mentor adquirí el hábito de tomar notas en todo momento - aún no lo logro por completo-, a pesar de que, al principio, lo consideraba una tarea sin sentido. Cuando me tocó hacerme cargo del primer grupo de manera formal, dimensioné las ventajas de tomar notas sobre lo que los estudiantes dicen, los acuerdos, las dudas que emergen, etc. (2019, p. 7-8)

Finalmente, en la etapa autonomía asistida, el profesor con experiencia se separa del profesor novel; solo lo acompaña a distancia para aclarar dudas, compartir, brindar apoyo. En esta nueva fase, el profesor novel coordina de modo independiente un grupo; ahora cuenta con 
José Antonio Serrano Castañeda | Noemí Hernández Suárez | Juan Mario Ramos

un mentor al que puede recurrir y un modelo de trabajo con el que pudo sentirse $-\mathrm{O}$ noidentificado a lo largo de los primeros dos momentos. Según Brockbank y McGill,

El profesor o profesora principiante, que aún puede estar intentando encontrar su sitio en el campus, no suele hallarse en condiciones de dar un paso por su cuenta y, con frecuencia, agradece que su mentor le proporcione un enfoque estructurado. El simple hecho de que el mentor les dedique con regularidad un momento semanal para darles información, consejo docente y el apoyo de una relación directa puede suponer una diferencia enorme para los nuevos profesores y profesoras. (2008. p.284)

El diálogo reflexivo entre ambos profesores (mentor y aprendiz) se convierte en una herramienta de trabajo imprescindible para ellos. Oliva narra:

\begin{abstract}
Me acerqué a los mismos profesores experimentados que han estado desde el inicio de mi aventura dentro de la universidad. Ellos me sugirieron prácticas puntuales como: tener todo por escrito y a la mano - acuerdos, correos enviados, respuestas-; sacar fotografías de los trabajos que los estudiantes entregaban, sistematizar sus entregas de tareas, actividades en clase, asistencias. Además, me orientaron sobre los trabajos o lecturas que podía abordar, los contactos con los que debía de hablar para fortalecer su formación, la manera de evaluar (continua, constante, con retroalimentaciones puntuales, mediante diversas estrategias y trabajos, entre ellos el diario individual y la trayectoria escolar). (2019, p.7)
\end{abstract}

Además, para este momento, se evidencia cómo las profesoras noveles ponen en juego los saberes, los tipos de práctica, así como los modelos de enseñanza trasmitidos y modelados por parte de los profesores experimentados. La transmisión supone un espacio abierto a la imaginación - en este caso - del profesor novel en el que puede desplegar sus potencialidades, poner en juego sus intuiciones, movilizar saberes — de diverso orden-, así como construir nuevas relaciones, significados y sentidos con la docencia, el acompañamiento, el acto educativo. Las profesoras noveles nos comparten al respecto:

Intento poner en acto las prácticas, estrategias y dispositivos que mis mentores me transmitieron de forma directa o que pusieron en juego con estudiantes, los cuáles observé y me apropié (cómo abordar una clase; cómo preparar las sesiones; cómo construir el programa de estudio del semestre; cómo elegir textos para cumplir con ciertos objetivos, cómo cuestionar, qué tipo de lecturas proponer). En el caso de los estudiantes de prácticas profesionales, al estar en un espacio real de trabajo, requieren de habilidades generales de la vida que se obtienen de experiencias que carecen. En este sentido, cuando tienen dudas sobre cómo resolver alguna dificultad en el espacio de prácticas, prefiero plantearles preguntas antes de sugerirles soluciones o explicaciones. He descubierto que, aunque me agradan las formas de enseñar de mis mentores y recupero gran parte de ellas, me diferencio en algunos aspectos debido a mi propia personalidad. (PRUSIA, 2019, p. 6). 
José Antonio Serrano Castañeda | Noemí Hernández Suárez | Juan Mario Ramos

En este mismo tenor Olivia advierte:

\begin{abstract}
Estar cerca de un profesor experimentado ha significado insertarme a una nueva cultura académica de una forma más afable. Desde saber con quién o cómo dirigirme para entregar algún tipo de documento; la forma de proceder para apoyar a algún estudiante en una determinada situación; las áreas con que la universidad cuenta para ser aprovechadas en la formación de docentes y estudiantes; los congresos, revistas, simposios que existen para escribir y compartir lo que se hace; hasta las maneras de ser en este ambiente como el ser responsable, profesional, colaborador, organizado, inquieto por proponer, innovar, sugerir y no tomarse nada de manera personal. Comprendo que si logro desarrollar estas formas de ser y proceder dentro de las diversas facetas de la cultura académica, en la docencia se vuelve algo implícito y de inspiración para los estudiantes, tal y como sucedió conmigo al ser comentora. (OLIVA, 2019, p.7)
\end{abstract}

Si bien, las profesoras noveles han desarrollado habilidades en torno a la docencia y se encuentran implicadas con una práctica reflexiva, también se han enfrentado a algunos retos, dificultades, inseguridades, desencantos. Ingresaron a la universidad con un ideal de la docencia y el trabajo académico; sin embargo, este se ha transformado después de haber vivido diferentes experiencias en el plano pedagógico, administrativo, institucional, etc.

Cuando cursaba la licenciatura brotó dentro de mí el deseo por dedicarme a la Educación Superior, una meta que, en definitiva, percibía lejana. Me cautivó el trabajo académico: la docencia, la investigación, la construcción de conocimiento. Ahora, después de un año y medio de estar dentro del contexto universitario he vivido decepciones de diversa índole: los estudiantes no me toman enserio (al menos eso he percibido); no he sido bien recibida por la planta docente de la UPN; el trabajo que realizo actualmente es inversamente proporcional a mi sueldo; los alumnos entran a clases no por una convicción de aprender e intimar con el conocimiento sino por razones completamente alejadas de esto; hay procesos administrativos engorrosos y burocráticos que "quitan mucho tiempo". Sin embargo, aún aviva en mi la convicción de dedicarme a la educación. Quizá soy una idealista con "harta" esperanza. No lo sé. Aún me falta mucho por aprender y estoy dispuesta a ello. Así como he tenido decepciones, también he vivido momentos de satisfacción personal. (PRUSIA, 2019, p. 20)

La profesora Oliva comparte las dudas que la invadían como profesora novel, a pesar de haber dado clases en primaria durante varios años antes de su afiliación a la educación superior;

El haber salido de mi zona de confort (la docencia en la educación básica), y enfrentarme a un ambiente nuevo, desconocido, ocasionó que me confrontara con miedos, inseguridades, paradigmas que no tenía del todo conscientes; los fui identificando en el transcurrir del tiempo con el apoyo de la escritura de mi diario individual y en pláticas o conversaciones con allegados.

Tenía varios miedos e inseguridades; diversas dudas pasaban por mi mente: ¿y si no estoy lo suficientemente preparada para ello?, ¿y si me preguntan de 
temáticas que aún no sé?, ¿y si me faltan habilidades para conducir a un grupo a nivel superior? La inseguridad y los temores se incrementaban cuando estudiantes llegaron a preguntarme si realizaba ahí su servicio social. Tener la edad cercana a algunos de ellos, e inclusive, llegar a ser más joven que otros, me hacía titubear sobre mi capacidad de conducirlos. Suponía que ellos o mis compañeros docentes dudaban de mí, cuando en realidad la única que lo hacía era yo misma. $(2019$, p. 3$)$

Como se advierte, la incursión al ámbito de la docencia universitaria supone una inmersión a prácticas institucionales específicas, por lo tanto, el acompañamiento formativo que reciban las profesoras noveles influirá en el modelamiento del "yo". Oliva reflexiona en torno a las implicaciones que tuvo su ingreso como profesora a la universidad:

Al ingresar al ámbito universitario, suponía que ser docente de este nivel significaba un valor mayor que en otros niveles educativos. Ahora no lo veo así. Es una cultura con sus particularidades y características. No dudo que varios profesores piensen como solía pensar yo. Se nota en sus formas de dirigirse o de ser con otros: en su prepotencia, su ego, o en la sobrevaloración de los títulos académicos por encima del trato humano. Ahora distingo que la cultura académica universitaria, como en otras, exige estarse preparando constantemente para la docencia. También para poner en marcha actividades adicionales como publicar lo que se hace, piensa o se indaga; gestionar programas o proyectos nuevos que impacten a la comunidad estudiantil; divulgar con otros sectores de la sociedad lo que se trabaja; coadyuvar en la titulación de los que concluyen con sus estudios universitarios. La cultura universitaria es distinta a las que le anteceden y reconocerla, aprender, actuar en ella es un aprendizaje del día a día. (OLIVA, 2019, p. 7)

Las profesoras noveles han dotado de sentido a su hacer docente a partir de las experiencias vividas dentro de un marco contextual en el que interactúan con otros. Hemos recortado el proceso de acompañamiento a las labores de docencia. Un proceso de inmersión diferenciada está en las actividades presentes en la labor de investigación, que merece un espacio aparte.

\section{Reflexiones finales}

«la educación es la construcción y la reconstrucción de historias personales y sociales» (Conelly, y Clandinin, 1995, p. 12);

Sin duda, la docencia en cualquier nivel escolar es compleja. La tarea de los profesores es ardua e impredecible; exige una actitud flexible y reflexiva en, durante y sobre la práctica. Muchos de los profesores novel conocen «a profundidad» una disciplina de conocimiento específico, así como modelos de enseñanza-aprendizaje y principios pedagógico-didácticos; sin embargo, 
carecen de competencias propias de la docencia que se adquieren con la práctica, a lo largo de la trayectoria profesional. De acuerdo con Schön

El conocimiento en la práctica se ejercita en los ámbitos institucionales propios de la profesión, y se organiza en función de sus unidades características de actividad y sus formas familiares de situaciones de la práctica, y se ve impedida o facilitado por su tronco común de conocimiento profesional y por su sistema de valores. (1992, p. 42)

No basta con promover procesos de acompañamiento a aquellos profesores inexpertos en el ámbito universitario por parte de profesores con experiencia, es esencial avivar en los nuevos profesores la voluntad por sistematizar su experiencia (que involucra diferentes esferas de la vida total de los sujetos: emociones, inquietudes, cuestionamientos, sentimientos, frustraciones, propuestas, percepciones de la institución) a través de la escritura constante en un diario reflexivo. Consideramos que esta práctica es trascendental en la vida de cualquier profesional de la educación, al permitirle "realizar una autopresentación de quién es y cómo ha evolucionado en su vida, efectuando un repaso por los momentos y circunstancias que le han puesto un especial impacto o han motivado un viaje en su trayectoria" (Bolívar, Domínguez y Fernández, 2001, p. 180).

El proceso de acompañamiento comprende dos elementos clave que se interconectan mutuamente: modelaje y transmisión. Los profesores con experiencia deben ser cuidadosos de no caer en actitudes prescriptivas o directivas; se trata de que pongan sobre la mesa aquellos asuntos, propuestas, orientaciones que, desde su posición de experto, consideran viables; las profesoras noveles son quienes decidirán lo que reconozcan viable de retomar a través de un ejercicio crítico y reflexivo, son agentes activos con capacidad de negatricidad (Ardoino, 1997). La mayoría de las dificultades que suelen enfrentar los profesores iniciados no están, necesariamente, relacionadas con los contenidos; también con aquellos sucesos impredecibles que se viven en una clase, en la institución, en el campo.

El encuentro entre profesor con experiencia y profesor novel, supone una transmisión de modos de ser y hacer que son (inter)operados por cada uno de los agentes a partir de marcos de referencia propios. En este sentido, el encuentro implica responsabilidad y, aunque en estos casos recuperados el acompañamiento fue vivido de modo acogedor no siempre sucede así; pueden vivirse desencuentros.

Por lo común, las ideas que tenemos sobre nuestro hacer no siempre resultan como habían sido proyectadas. Así, es sensato - en tanto primero humanos y después profesoresvivir miedo, frustraciones, decepciones; la tarea, entonces, es reconocerlos, aceptarlos, hacerlos 
parte de la práctica, reflexionar sobre ellos y tomar decisiones. Empero, no tendría por qué vivirse este cúmulo de emociones y acontecimientos en solitario; pueden ser socializadas y revisar qué se puede hacer al respecto. No se trata de reconocer solo lo negativo, lo que no se hizo, también los logros, lo que sí se hizo. En este caso vale hacer consciente los métodos, las inclinaciones, las filias, los sentimientos; sustancias que alimentan nuestras decisiones y la forma en cómo nos paramos en el mundo.

Somos conscientes que el acompañamiento que recibe el profesor novel está sostenido por un ideal de profesor que requiere la educación superior, un profesional reflexivo y comprometido con su formación; preparado para modelar con los estudiantes a los que atenderá, la propia práctica reflexiva de la que es parte. Creemos que, si queremos modificar las tradicionales prácticas de enseñanza-aprendizaje, vale iniciar con los profesores de recién ingreso a la institución, quienes sucederán a los que ya están dentro del ámbito de la educación superior; pensamos en una cadena entre predecesores y sucesores que se proponga un cambio real en las prácticas institucionales, no solo como aspiración de innovación sino como habitus reflexivo sobre transformación institucional.

Reconocemos que este tipo de procesos se enfrentan a circunstancias que consiguen frenar su continuidad y que no tienen que ver con la actitud de los estudiantes: la falta de recursos económicos por parte de la institución; la ausencia de profesores con experiencia dispuestos a acompañar a profesores novel; prácticas docentes viciadas. Mantenemos la confianza en que las experiencias de acompañamiento al interior de la UPN podrán constituirse como uno de los varios marcos de referencia que ya hay en torno al tema, que invite a pensar en nuevas rutas de acción, consideremos lo que Suárez ha planteado,

Una vez que estos relatos de experiencia circulan y son conocidos en comunidades de docentes lectores-escritores de narraciones pedagógicas y se vuelven públicamente disponibles, esas historias de enseñanza se constituyen en "documentos pedagógicos" e intervienen en el debate público y especializado de la educación. (2015, p.97)

Idea en consonancia con la aspiración de Dewey relativa a la conformación de una ciencia de la educación con base casuística.

Una permanente sucesión de informes minuciosos, extraídos de casos reales, que den cuenta de las condiciones que en la experiencia han demostrado ser favorables o desfavorables para el aprendizaje, podría revolucionar por completo el tema del método... El progreso de cualquier ciencia de la educación depende precisamente de la acumulación sistemática de este tipo de material [Dewey, 1974, pág. 181]. (SCHÖN, 1992, p. 273) 
Por otro lado, cabe dejar abierto el tema de la experiencia de transmisión. No podemos olvidar que la transmisión es "la acción de un espíritu sobre otro" (STEINER, LASDJALI, 2006,1997, p. 24) y como proceso de intercambio "El profesor ha de sacar al alumno de su mundo, conducirle hasta donde no habría llegado nunca sin su ayuda, y traspasarle un poco de su alma, porque quizá toda formación no sea más que una deformación” (STEINER, LASDJALI, 2006, p. 37).

En la transmisión se pone en juego la vida de los sujetos. Si bien es cierto que el encuentro es actual, en el proceso de acompañamiento de profesores novel y con experiencia están las historias sedimentadas (imagos) que los actores sociales han construido activamente - en la vida en las aulas. Son los profesores memorables. Noción que "hace referencia a aquellos docentes que han dejado una huella en nuestra biografía escolar, o bien se han convertido en referentes del campo por su trayectoria y su profesionalismo (PORTA, AGUIRRE, BAZAN, 2017, p. 20).

La huella dejada por estos profesores se constituye como un referente para la acción futura. Las experiencias vividas por el profesor novel en su relación con otros docentes forman parte del relato que construye en torno a lo escolar, lo educativo, lo institucional. Lo que se pone en juego en la interacción entre profesor novel y experimentado es un intercambio de prácticas, saberes, valores, normas, representaciones, creencias, integradas y resignificadas a través de un ejercicio de reflexión.

Los saberes transmitidos por parte del profesor con experiencia son relaborados por el profesor novel a partir del acervo experiencial que posee y enviados a una base de imaginación que lo impulsan a indagar, experimentarse en un futuro, crear nuevas relaciones. En este sentido, el hecho transmisivo - en este caso, desde un proceso de acompañamiento — involucra transformación, más que pura conservación o reproducción (MURGA, 2017).

En la transmisión está la cultura en acto, lo heredado y por heredarse. Como advierte Bárcena, «Hay toda una estética y toda una poética de la relación maestros y discípulos» (2018, p. 75). Tema que tiene larga data en las reflexiones pedagógicas pero que se ha visto oscurecido por "capitalismo cognitivo" (BÁRCENA, 2018, p. 75) imperante en nuestro medio que, con pretensiones científicas, olvida que en todo acto humano la imaginación creadora está en juego.

\section{REFERENCIAS}

ARDOINO, J. Conferencia impartida en el Centro de Estudios sobre la Universidad en UNAM, México el 4 de noviembre de 1997. Recuperado de file:///C:/Users/MyPC/Downloads/La\%20implicacion\%20\%20-\%20Ardoino.pdf 
ARNAU, J. Historia de la imaginación. Del antiguo Egipto al sueño de la ciencia. Barcelona: Espasa, 2020.

BÁRCENA, F. Maestros y discípulos. Anatomía de una relación. Teoría de la educación. Revista interuniversitaria, núm 30, 2, p. 73-108, 2018.

BERNSTEIN, R. Filosofía y democracia: John Dewey. Barcelona: Herder, 2010.

BOLÍVAR A., DOMINGO J. Y FERNÁNDEZ, M. La investigación biográfico-narrativa en educación. Enfoque y metodología. Madrid: La Muralla, 2001.

BOURDIEU, P. Razones prácticas. Sobre la teoría de la acción. Barcelona: Anagrama, 1997.

BOURDIEU, P.; WACQUANT, J. D. Respuestas por una antropología reflexiva. Ciudad de México: Grijalbo, 1995.

BROCKBANCK, A. Y MCGILL, I. Aprendizaje reflexivo en la educación superior. Madrid: Morata, 2008.

CONNELLY, M.; CLANDININ, D. J. Relatos de experiencia e investigación narrativa. En: LARROSA, J. et al. Déjame que te cuente: Ensayos sobre narrativa y educación, p. 11-59. Barcelona: Laertes, 1995.

CONTRERAS, J. Relatos de experiencia, en busca del saber pedagógico. Revista Brasileira de Pesquisa (Auto) Biográfica, Salvador Vol. 1, №1, p. 14-30, 2016. BIOgraph e Editores RBPAB. Disponível em: 10.31892/rbpab2525-426X.2016.v1.n1.p14-30, Acesso em: 01, octubre, 2020.

DA CONCEIÇÃO, M. Narrativa, experiencia y reflexión auto-biográfica: por una epistemología del sur en educación. En MURILLO, G. J. (comp.). Narrativas de experiencia en educación y pedagogía de la memoria. Buenos Aires: FFyLUBA, 2015.

DEBRAY, R. Transmitir. Buenos Aires: Manantial, 1997.

DELORY-MOMBERGER, CH. Biografía de educación. Figuras del individuo-proyecto. Buenos Aires: FFyL-UBA-CLACSO, 2009.

DEWEY, J. Democracia y educación. Una introducción a la filosofía de la educación. Madrid: Morata, 1998.

DEWEY, J. El arte como experiencia. Barcelona: Paidós, 2008.

LEYVA, G.; KOZLAREK, O. Introducción. En Joas, H. Creatividad, acción y valores. Hacia una sociología de la contingencia. Ciudad de México: UAM-Goethe Institut-DAAD, 2002.

MUNNÉ, F. La interacción social. Teorías y ámbitos. Barcelona: PPU, México, 1995. 
MURGA. M.L. (Coord.) Dogmas de la educación. Irrumpir la Uniformidad. Ciudad de México: UPN, 2017.

PERRENAUD, P. Desarrollar la práctica reflexiva en el oficio de enseñar. Profesionalización y razón pedagógica. Barcelona: Graó-Colofón, 2011.

PORTA, L. y RAMALLO, F. La investigación narrativa en educación: experiencia, escritura de sí y (auto)biografía. Diálogo con José Antonio Serrano Castañeda. Revista de Educación, año IX, no. 13, p. 209-222, 2018.

PORTA, L.; AGUIRRE J.; BAZAN, S. La práctica docente en los profesores memorables. Reflexividad, narrativa y sentidos vitales, Diálogos Pedagógicos, año XV, No 30, p. 15-36. octubre 2017.

RUFINETTI, E. Anticipación y diálogo en Mijaíl Bajtín y Hans Gadamer. Un intento de 'mediación'. Tópicos, núm. 25, p. 40-62 Universidad Católica de Santa Fe, Santa Fe, 2013.

SCHÖN, D. La formación de profesionales reflexivos. Hacia un nuevo diseño de la enseñanza y el aprendizaje de las profesiones. Barcelona: Paidós-MEC, 1992.

SERRANO, J. A. Interaccionismo Simbólico. En SALMERÓN, A.M.; TRUJILLO, B.F.; RODRÍGUEZ, A.; DE LA TORRE, M. Diccionario Iberoamericano de Filosofía de la Educación. 2016. En http://fondodeculturaeconomica.com/dife/definicion.aspx ?l=I\&id=81.

SERRANO, J.A. Y RAMOS, J.M. Narrar a vida: deliberações no campo biográfico. En MARTINS, R.; TOURINHO, I.; SOUZA, E.C. (Coords.). Pesquisa narrativa: interfases entre histórias de vida, arte e educaçao. Santa Maria: Ed. da UFSM, 2017. p. 75-97.

STEINER, G.; LADJALI, C. Elogio de la transmisión. Madrid: Siruela, 2006.

SUÁREZ, D. Relatos de experiencia, redes pedagógicas y prácticas docentes: documentación narrativa de experiencias escolares en el nivel inicial. En: MURILLO, G. J. (comp.).

Narrativas de experiencia en educación y pedagogía de la memoria. Buenos Aires: FFyLUBA, 2015.

TODOROV, T. La vida en común. Ensayos de antropología general. Madrid: Taurus. 1995.

VAN MANEN, M. Investigación educativa y experiencia vivida. Ciencia humana para una pedagogía de la acción y de la sensibilidad. Barcelona: Idea Books. 2003.

\section{Documentos personales}

Oliva. Diario personal. 2017.

Oliva. Diario personal. 2019.

Prusia. Diario personal, 2019. 


\section{SOBRE OS AUTORES:}

\section{José Antonio Serrano Castañeda}

Doctor en Pedagogía por la Universidad de Barcelona. Profesor Titular de la Universidad Pedagógica Nacional-Ajusco, México. Investigador SNI-1 Conacyt y profesor PRODEP. Integrante del cuerpo académico consolidado Prácticas institucionales y constitución del sujeto de la educación (CAC PICSE). Es responsable del grupo de investigación dedicado a la reflexión sobre lo biográfico y la narrativa en educación SPECE (Sujetos y prácticas educativas en contextos escolares). Ha participado en diferentes proyectos de investigación, así como de formación de docentes en el sistema educativo mexicano. Ha publicado diversos artículos sobre teoría educativa, curriculum, formación de docentes, alfabetización académica y el campo biográfico-narrativo. Es responsable de la Maestría en Desarrollo Educativo, programa consolidado en las políticas de CONACYT. Líneas de investigación: curriculum, formación docente, investigación narrativa, literacidad académica. E-mail: jserrano@g.upn.mx

(iD https://orcid.org/0000-0002-7272-7001.

\section{Noemí Hernández Suárez}

Licenciada en Pedagogía por la UPN. Profesora Asociada M/T. Integrante de la Red SPECE (Sujetos y prácticas educativas en contextos escolares). Estudiante de la Maestría en Desarrollo Educativa, Línea Prácticas Institucionales y Formación Docente.

Ha desarrollado investigación en torno a la educación artística y colaborado en proyectos de prácticas de acompañamiento y literacidad académica en educación superior. En el ámbito de las prácticas profesionales se ha desempeñado como mentora en espacios de prácticas profesionales de la Licenciatura Administración Educativa. Ha participado en alguno congresos nacionales e internacionales. E-mail: nsuarez@upn.mx

(iD https://orcid.org/0000-0003-1731-1465

\section{Juan Mario Ramos Morales}

Doctor en Ciencias Administrativas por el Instituto Tecnológico y de Estudios Superiores de Monterrey, Campus Ciudad de México. Profesor Titular de la Universidad Pedagógica Nacional-Ajusco, México. Investigador SNI-1 Conacyt y profesor PRODEP. Integrante del cuerpo académico consolidado Prácticas institucionales y constitución del sujeto de la educación (CAC PICSE) y de la Red SPECE (Sujetos y prácticas educativas en contextos escolares). Ha participado en diferentes proyectos de investigación y en la gestión de programas de posgrado. Ha publicado diversos artículos sobre el campo biográfico-narrativo en educación. Líneas de investigación: prácticas profesionales, prácticas de formación, prácticas de acompañamiento, investigación biográfico-narrativa. E-mail: jramos@g.upn.com

iD https://orcid.org/0000-0002-5364-1827 